UDK 517

R. F. Shamoyan

\title{
ON DECRIPTIONS OF CLOSED IDEALS OF ANALYTIC AREA NEVANLINNA TYPE CLASSES IN A CIRCULAR RING ON A COMPLEX PLANE $\mathbb{C}$
}

\begin{abstract}
We define certain new large area Nevanlinna type spaces in circular ring $K$ on a complex plane and provide complete decriptions of ideals of these new scales of spaces. Our results extend some previously known assertions.
\end{abstract}

Key words: analytic Area Nevanlinna, closed ideals

\section{$\S 1$. Introduction}

Assuming that $\mathbb{D}=\{z \in \mathbb{C}:|z|<1\}$ is the unit disk of the finite complex plane $\mathbb{C}, \mathrm{T}$ is the boundary of $\mathbb{D}$ and $H(\mathbb{D})$ is the space of all functions holomorphic in $\mathbb{D}$, introduce the following classes of functions:

$$
\widetilde{N}_{\alpha}^{\infty}=\left\{f \in H(\mathbb{D}): T(\tau, f) \leq C_{f}(1-\tau)^{-\alpha}, \quad 0 \leq \tau<1\right\}, \quad \alpha \geq 0,
$$

where $T(\tau, f)$ is Nevanlinna's characteristic (see eg. [1, 2]). It is obvious that if $\alpha=0$, then $\widetilde{N}_{0}^{\infty}=N$, where $N$ is the well-known Nevanlinna's class. The following statement on parametric representation in spaces in unit disk holds by Nevanlinna's classical result on the parametric representation of $N$ (see eg. [1,2]) and it serves (see [1]) as a base of all theory on area Nevanlinna type spaces. The $N$ class coincides with the set of functions representable in the form

$$
f(z)=C_{\lambda} z^{\lambda} B\left(z,\left\{z_{k}\right\}\right) \exp \left\{\int_{-\pi}^{\pi} \frac{d \mu(\theta)}{1-z e^{-i \theta}}\right\}, \quad z \in \mathbb{D},
$$

where $C_{\lambda}$ is any complex number, $\lambda$ is any nonnegative integer, $B\left(z,\left\{z_{k}\right\}\right)$ is the classical Blaschke product with zeros $\left\{z_{k}\right\}_{k=1}^{+\infty} \subset \mathbb{D}$ enumerated according their multiplicities and satisfying the condition $\sum_{k}\left(1-\left|z_{k}\right|\right)<+\infty$ and $\mu(\theta)$ is any function of bounded variation in $[-\pi, \pi]$.

(C) Shamoyan R. F., 2012 
Later this important result was extended to weighted Nevanlinna spaces we defined above and other similar spaces for example to so-called Nevanlinna-Djrbashyan spaces (see eg. $[1,2,3,8,17]$ ). The goal of this paper is to continue our earlier investigation on closed ideals started in [5] in certain new large analytic area Nevanlinna type spaces in the unit disk there and in this paper mentioned type parametric representations are playing crucial role. We obtain similar descriptions of closed ideals, but in circular rings. The problem of description of closed ideals in various spaces of analytic and entire functions were considered by many in $[4$, $6,7,9,10,11,12]$. Papers $[11,12]$ are closest to this investigation. They provide first descriptions of closed ideals of area Nevanlinna type spaces in the unit disk. Our intention here also is to extend these and some results from $[8,14]$ on closed ideals in spaces in the circular ring to larger spaces of area Nevanlinna type in the same circular ring. Note in our previous paper [3] we provided already such an extention procedure in the unit disk, extending some known classical results about zero sets in certain analytic Nevanlinna spaces from [1] and from [2] to mentioned larger spaces in unit disk. For formulation of our theorems we need to introduce first these new analytic spaces in the unit disk. We define below three different scales of large area Nevanlinna spaces in the unit disk and then based on that in circular ring. These large spaces in unit disk appeared and studied for the first time in $[3,5]$. We note finnaly we provide some direct applications of our recent results on parametric representations in spaces in circular rings from [17]. We also mention a separate paper [6] where complete analogues of spaces we consider in this note in case of upperhalfplane were considered by authors and descriptions of zero sets for them was given.We denote below as usual by $d m_{2}(z)$ the normalized Lebegues measure in the unit disk on the complex plane. Let further

$$
\begin{aligned}
& N_{\alpha, \beta}^{p}=\{f \in H(\mathbb{D}): \\
& \left.\int_{0}^{1}\left[\int_{|z| \leq R} \ln ^{+}|f(z)|(1-|z|)^{\alpha} d m_{2}(z)\right]^{p}(1-R)^{\beta} d R<+\infty\right\}, \\
& N_{\alpha, \beta_{1}}^{\infty}=\{f \in H(\mathbb{D}): \\
& \left.\sup _{0 \leq R<1}\left[\int_{|z| \leq R} \ln ^{+}|f(z)|(1-|z|)^{\alpha} d m_{2}(z)\right](1-R)^{\beta_{1}}<+\infty\right\},
\end{aligned}
$$

where it is assumed that $\beta_{1} \geq 0, \alpha>-1, \beta>-1$ and $0<p<\infty$. Note that various properties of $N_{\alpha, 0}^{\infty}$ are studied in [2]. In particular, 
both books $[1,2]$ provide complete description of zero sets and parametric representations of $N_{\alpha, 0}^{\infty}$. Later these assertions were extended by us to larger analytic spaces we defined above in [3] and these results in his turn were used in [5].

Thus, generally speaking it is natural to consider the problem on extension of various known results, for example, to all $N_{\alpha, \beta_{1}}^{\infty}$ classes.

We assume also in addition that

$$
\begin{aligned}
& (N A)_{p, \gamma, v}=\{f \in H(\mathbb{D}): \\
& \left.\int_{0}^{1}\left[\sup _{0<\tau<R} T(f, \tau)(1-\tau)^{\gamma}\right]^{p}(1-R)^{v} d R<+\infty\right\},
\end{aligned}
$$

where $\gamma \geq 0, v>-1$ and $0<p<\infty$, and also

$$
\begin{aligned}
& N_{\alpha, \beta}^{\infty, p}=\{f \in H(\mathbb{D}): \\
& \left.\sup _{0 \leq R<1} \int_{0}^{R}\left[\int_{\mathrm{T}} \ln ^{+}|f(|z| \xi)| d \xi\right]^{p}(1-|z|)^{\alpha} d|z|(1-R)^{\beta}<+\infty\right\},
\end{aligned}
$$

where $0<p<\infty, \alpha>-1$ and $\beta \geq 0$. Note that the zero sets of $N_{\alpha, \beta}^{\infty, p}$ are described in [9] for $\beta=0$ and later for all positive values of $\beta$ in [3].

It is not difficult to verify that all the above mentioned analytic classes are topological vector spaces with complete invariant metrics see for this, for example, [5].

Throughout the paper, we write $c$ to denote a positive constant which is independent of the functions or variables being discussed.

The following assertion from [1] is crucial for our investigation.Let $\left(z_{k}\right)$ be an arbitrary sequence of complex numbers from the unit disk $D$, so that the following condition holds

$$
\sum_{k=1}^{+\infty}\left(\left(1-\left|z_{k}\right|\right)^{\beta+2}\right)<+\infty
$$

$\beta>-1$, then the Djrbashian infinite product (see [1])

$$
\prod_{\beta}\left(z, z_{k}\right)
$$

is an analytic function and converges uniformly in D and have zeros only at mentioned sequence $\left(z_{k}\right)$. 
Complete descriptions of closed ideals of classes we defined above in case of unit disk were given in [5]. The intention of this paper to expand further these results to the case of circular rings. The theory of analytic function spaces in circuar rings was developed before partially in [13, 15]. We mention here also $[8,14]$ where similar problems discussed in this paper were considered and solved for other analytic area Nevanlinna type spaces in circular rings. Classes we introduce in circular rings are larger and our theorems can be considered as extentions of some previously known results from $[8,14]$.

For $0<R_{1}<R_{2}<+\infty$ we denote by $\mathrm{K}$ the usual circular ring on the complex plane $K=\left\{z \in C, R_{1}<|z|<R_{2}\right\}, K=K\left(R_{1}, R_{2}\right)$, we also denote by $H(K)$ the space of analytic functions in this circular ring and for $f$ function so that $f \in H(K)$ we denote the Nevanlinna characteristic in circular ring of $f$ by

$$
T(\tau, f) .
$$

We also use the following definition. We denote by $Z(f)$ the set of zeros of $f$ function in a circular ring we defined above. Let $r_{0}=\frac{\left(R_{1}+R_{2}\right)}{2}$, by $Z\left(R_{1}, r_{0}, f\right)$ we denote all those points from circular ring $K\left(r_{0}, R_{1}\right)$ which at the same belong to $Z(f)$. Similarly we define $Z\left(R_{2}, r_{0}, f\right)$. Let further $Z\left(R_{1}, r_{0}, f\right)=\left(z_{k}\right), Z\left(R_{2}, r_{0}, f\right)=\left(w_{k}\right)$.

We define new spaces in circular ring $K$. Shortly speaking the idea is to replace in quazinorms of spaces we provided above the integration interval unit interval $(0,1)$ by $\left(R_{1}, R_{2}\right)$ and the other interval of integration which appeared there $(0, R)$ by $\left(R_{1}, R\right)$. The conditions on parametrs are the same. Let

$$
\begin{aligned}
& (N A)_{p, \gamma_{1}, \gamma_{2}, v_{1}, v_{2}}=\{f \in H(\mathbb{K}): \\
& \left.\int_{R_{1}}^{R_{2}}\left[\sup _{R_{1}<\tau<R} T(f, \tau)(R-\tau)^{\gamma_{1}}\left(\tau-R_{1}\right)^{\gamma_{2}}\right]^{p}\left(R-R_{1}\right)^{\beta_{1}}\left(R_{2}-R\right)^{\beta_{2}} d R<+\infty\right\},
\end{aligned}
$$

we will write $f \in(N A)_{p, \gamma_{1}, v_{1}}$ meaning the quazinorm we provided above without factors which contain $\gamma_{2}$ and $v_{2}$ and in the same way we will use the notation $(N A)_{p, \gamma_{2}, v_{2}}$ meaning the quazinorm we provided above, but without those factors which contains $\gamma_{1}$ and $v_{1}$. Moreover in analytic spaces in circular ring $K$ which we will define below the same will be assumed. We define $(N)_{\alpha_{1}, \alpha_{2}, \beta_{1}, \beta_{2}}^{\infty, p}$ as space of analytic functions in $K$ 
with finite quazinorm

$\sup _{R_{1}<R<R_{2}}\left[\int_{R_{1}}^{R} T(f,|z|) \mid(R-|z|)^{\alpha_{1}}\left(|z|-R_{1}\right)^{\alpha_{2}} d m_{-2}(z)\right]^{p}\left(R-R_{1}\right)^{\beta_{1}}\left(R_{2}-R\right)^{\beta_{2}}$ that

and $(N)_{\alpha_{1}, \alpha_{2}, \beta_{1}, \beta_{2}}^{p}$ as space of functions analytic in circular ring $K$ so

$\int_{R_{1}}^{R_{2}}\left[\int_{R_{1}}^{R} T(f,|z|)\left(|z|-R_{1}\right)^{\alpha_{2}}(R-|z|)^{\alpha_{1}} d m_{2}(z)\right]^{p}\left(R-R_{1}\right)^{\beta_{1}}\left(R_{2}-R\right)^{\beta_{2}} d R<+\infty$.

\section{$\S 2$. Theorems on Closed Ideals of $N_{\alpha_{1}, \alpha_{2}, \beta_{1}, \beta_{2}}^{p}$, $(N A)_{p, \gamma_{1}, \gamma_{2}, v_{1}, v_{2}}$ and $N_{\alpha_{1}, \alpha_{2}, \beta_{1}, \beta_{2}}^{\infty, p}$ Classes in Circular \\ Rings}

In this section we formulate our main results. These are three theorems providing descriptions of closed ideals for each space defined above. These results can be considered as direct extentions of our earlier theorems in the unit disk from [5] and as applications of our recent results on parametric representations of analytic area Nevanlinna type classes we defined above in circular rings [17].

Theorem 1. Let $I$ be a closed ideal of topological algebra $(N)_{\alpha_{1}, \alpha_{2}, \beta_{1}, \beta_{2}}^{p}$ and $0<p<\infty, \alpha_{j}>-1, \beta_{j}>-1, j=1,2$, and $Z(I)$ is an intersection of all $Z(f)$ sets so that $f \in I$, where $Z(f)$ is a set of all zeros of $f$ in a circular ring $K$. Then

$$
I=\left\{f \in(N)_{\alpha_{1}, \alpha_{2}, \beta_{1}, \beta_{2}}^{p},(Z(f)) \supset(Z(I))\right\} .
$$

Let $E_{1} \subset K\left(R_{1}, r_{0}\right), E_{2} \subset K\left(r_{0}, R_{2}\right)$, be arbitrary two sets $E_{1}=\left(z_{k}\right)$, $E_{2}=\left(w_{k}\right)$ satisfying the following conditions

$$
\sum_{k=1}^{\infty} \frac{\left(n_{k}\right)^{p}}{2^{k\left(\beta_{1}+\alpha_{1} p+2 p+1\right)}}, \quad \sum_{k=1}^{\infty} \frac{\left(m_{k}\right)^{p}}{2^{k\left(\beta_{1}+\alpha_{1} p+2 p+1\right)}}
$$

where

$$
\begin{aligned}
& n_{k}=\left\{\operatorname{card} z_{k}:\left|z_{k}\right|>R_{1}\left(1+2^{-k}\right)\right\}, \\
& m_{k}=\left\{\operatorname{card} w_{k}:\left|w_{k}\right|<R_{2}\left(1-2^{-k}\right)\right\} .
\end{aligned}
$$

Then for each set $E$ which is a union of such two sets $E_{1}$ and $E_{2}$ the following set 


$$
I=\left\{f \in(N)_{\alpha_{1}, \alpha_{2}, \beta_{1}, \beta_{2}}^{p},(Z(f)) \supset E\right\}
$$

is a closed ideal of topological algebra $(N)_{\alpha_{1}, \alpha_{2}, \beta_{1}, \beta_{2}}^{p}$.

In the following two theorems we use both $n_{k}$ and $m_{k}$ in a same sense as above.

Theorem 2. Let I be a closed ideal of topological algebra $(N)_{\alpha_{1}, \alpha_{2}, \beta_{1}, \beta_{2}}^{\infty, p}$, and $\alpha_{j}>-1, \beta_{j}>0, j=1,2, p \in(0, \infty)$.

Let $Z(I)$ be an intersection of all $Z(f)$ sets so that $f \in I$, where $Z(f)$, is a set of all zeros of $f$ in a circular ring $K$.

Then

$$
I=\left\{f \in(N)_{\alpha_{1}, \alpha_{2}, \beta_{1}, \beta_{2}}^{\infty, p},(Z(f-)) \supset(Z(I))\right\} .
$$

Let $E_{1} \subset K\left(R_{1}, r_{0}\right), E_{2} \subset K\left(r_{0}, R_{2}\right)$, be arbitrary two sets $E_{1}=\left(z_{k}\right), E_{2}=$ $\left(w_{k}\right)$ satisfying the following conditions.

Let $n_{1}(\tau)=\left\{\operatorname{card} z_{k}:\left|z_{k}\right|>\tau\right\}, \tau \in\left(R_{1}, R_{2}\right)$, and for same $\tau$ we put $n_{2}(\tau)=\left\{\operatorname{card} w_{k}:\left|w_{k}\right|<\tau\right\}$. We assume that

$$
\left(n_{1}\right)^{p}(\tau)<c\left(\tau-R_{1}\right)^{-\alpha-\beta-p-1},
$$

and the same is true for $n_{2}$ with $\left(R_{2}-\tau\right)$ and with $\alpha_{2}$ and $\beta_{2}$ instead of $\alpha_{1}$ and $\beta_{1}$.

Then for each set $E$ which is a union of such two sets $E_{1}$ and $E_{2}$ the following set

$$
I=\left\{f \in(N)_{\alpha_{1}, \alpha_{2}, \beta_{1}, \beta_{2}}^{\infty, p},(Z(f)) \supset E\right\}
$$

is a closed ideal of topological algebra $(N)_{\alpha_{1}, \alpha_{2}, \beta_{1}, \beta_{2}}^{\infty,}$.

Theorem 3. Let I be a closed ideal of topological algebra $(N A)_{p, \gamma_{1}, \gamma_{2}, v_{1}, v_{2}}$, and $\gamma_{j}>0, v_{j}>-1, j=1,2, p \in(0, \infty)$.

Let $Z(I)$ be an intersection of all $Z(f)$ sets so that $f \in I$, where $Z(f)$ is a set of all zeros of $f$ in circular ring $K$.

Then

$$
I=\left\{f \in(N A)_{p, \gamma_{1}, \gamma_{2}, v_{1}, v_{2}},(Z(f)) \supset(Z(I))\right\} .
$$

Let $E_{1} \subset K\left(R_{1}, r_{0}\right), E_{2} \subset K\left(r_{0}, R_{2}\right)$ be arbitrary two sets $E_{1}=\left(z_{k}\right)$, $E_{2}=\left(w_{k}\right)$ satisfying conditions.

$$
\sum_{k=1}^{\infty} \frac{\left(n_{k}\right)^{p}}{2^{k\left(p \gamma_{1}+p+1+v_{1}\right)}}<\infty
$$


and the same condition is true for $m_{k}$ instead of $n_{k}$ and with $\gamma_{2}$ and $v_{2}$ instead of $\gamma_{1}$ and $v_{1}$.

Then for each set $E$ which is the union of such two sets $E_{1}$ and $E_{2}$ the following set

$$
I=\left\{f \in(N A)_{p, \gamma 1, \gamma_{2}, v_{1}, v_{2}},(Z(f)) \supset E\right\}
$$

is a closed ideal of topological algebra $(N A)_{p, \gamma_{1}, \gamma_{2}, v_{1}, v_{2}}$.

We remark finnaly that as an abstract these results we formulated above were provided before in [16].

\section{Biblyography}

[1] Djrbashyan M., Shamoyan F. Topics in the theory of $A_{\alpha}^{p}$ spaces // Leipzig, Teubner-Texte zur Mathematik. 1988. V. 105.

[2] Hedenmalm H., Korenblum B., Zhu K. Theory of Bergman spaces // Graduate Texts in Mathematics. V. 199. New York: Springer-Verlag, 2000.

[3] Shamoyan R., Li H. Descriptions of zero sets and parametric representations of certain new analytic area Nevanlinna type spaces in the unit disk // Kragujevac Journal of Mathematics. 2010. V. 34. P. 73-89.

[4] Korenblum B. Closed ideals in the ring $A^{n} / /$ Funkcion. Anal. i prilogeni. 1972. V. 3. P. 38-52 (In Russian).

[5] Shamoyan R., Li H. Characterizations of closed ideals and main parts of some analytic and meromorphic classes of area Nevanlinna type in the unit disk // International Journal of Mathematics and Statistics. 2011. V. 10. P. $109-121$.

[6] Rudin W. Closed ideals in an algebra of analytic functions // Canadian Journal of Mathematics. 1957. V. 9. P. 426-434.

[7] Estrele J., Stouse E., Zouakia F. Closed ideals of $A^{+}$and Cantor sets // Journal Reigne Angew. Math. 1994. 449. P. 65-79.

[8] Kursina I. Factorization and parametric representation of weighted spaces of analytic functions in the unit disk // Dissertation. Bryansk: Bryansk State University, 2000. (In Russian).

[9] Krasichkov I. On closed ideals in locally convex algebra of entire functions // Siberian Mathematical Journal. 1968. V. 9(1). P. 77-98. (In Russian).

[10] Shirokov N. Closed ideals of algebras of type $B_{p, q}^{\alpha} / /$ Izv. Acad. Nauk. SSSR. 1982. V. 6 46. P. 1316-1332. (In Russian).

[11] Roberts J., Stoll M. Prime and principal ideals of algebra $N^{+} / /$Archiv der Mathematik. 1976. V. 27. P. 387-393. 
[12] Shamoyan F. Description of closed ideals in area Nevanlinna-Djrbashian spaces and characterizartion of elements of $N^{+} / /$Izv.Acad. NAuk. Armenii. 1988. V. 6. P. 575-587. (In Russian).

[13] Zverovich V. On certain spaces of analytic functions in circular rings // MAt. Sbornik. 1956. V. 40. 82. P. 225-238. (In Russian).

[14] Kipen I. Parametric representatiions and closed ideals in certain spaces of analytci functions in circular rings // Proc. of conference. Mat.model.in hum. and natural sciences. VGU. 2000. P. 131. (In Russian).

[15] Kasyaniuk S. On functions of $A$ and $H$ spaces in cicular rings // Mat. Sbornik. 1957. V. 42. P. 301-326. (Russian)

[16] Shamoyan R. On some new spaces of analytic functions of area Nveanlinna type in circular ring // Proc. of Conference Harmonic analysis and approximation. Tsakhadzor. Armenia. 2011. (In English).

[17] Shamoyan R. On some parametric representations of new analytic area Nevanlinna type spaces in circular ring K // Preprint. 2012.

Bryansk State Technical University,

Department of Mathematics

Bryansk 241050, Russia.

E-mail: rshamoyan@gmail.com 